\title{
Validação de questionários de frequência alimentar: uma revisão sistemática
}

\section{Validation of food frequency questionnaire: a systematic review}

Thays de Ataide e Silval

Sandra Mary Lima Vasconcelos ${ }^{2}$

\footnotetext{
1 Programa de Pós-graduação em Nutrição. Departamento de Nutrição da Universidade Federal de Alagoas. Maceió, AL, Brasil.

2 Professora adjunta da Faculdade de Nutrição, Departamento de Nutrição da Universidade Federal de Alagoas. Maceió, AL, Brasil.

Correspondência / Correspondence Sandra Mary Lima Vasconcelos E-mail:sandra-mary@hotmail.com
}

\section{Resumo}

Objetivo: Revisar artigos originais que testaram a validade de questionários de frequência alimentar (QFA) para população adulta e/ou idosa, tendo como padrão de referência o inquérito dietético recordatório de 24 horas (IDR24H). Métodos: Foram consultadas as bases de dados eletrônicas SciELO, PubMed, LILACS, IBECS e MEDLINE, com as palavras-chave: validade (validity), reprodutibilidade (reproducibility) e questionário de frequência alimentar (food frequency questionnaire), utilizando o operador lógico AND. Resultados: Foram identificados 15 estudos que subsidiaram esta revisão com 52 a 222 itens alimentares, predominando o tipo quantitativo. Todos testaram a validade de QFAs com 2 a 16 IDR24H; 11 a reprodutibilidade, atingindo coeficientes de correlação entre 0,4 e 0,7 em cinco estudos $>0,7$ em dois $<0,4$ em quatro estudos e os demais dentro de uma faixa mais ampla, que foi de $<0,4$ a $>0,7$. O tamanho da amostra estudada variou entre 40 e 499 indivíduos. Oito artigos relataram que ao menos um IDR24H aplicado correspondia a pelo menos um dia de final de semana. Conclusóes: A maioria dos estudos apresentou resultado satisfatório para validade, sendo sugeridos pelos autores como ferramenta útil em estudos epidemiológicos, para populações semelhantes às do QFA de origem.

Palavras-chave: Validade. Consumo de Alimentos. Questionário. Adulto. Idoso. 


\section{Abstract}

Objective: To review original articles that tested the validity of food frequency questionnaires (FFQ) for adult and old population, with the reference standard of the 24-hour food recall (FR24H). Methods: We consulted the electronic databases SciELO, PubMed, LILACS, MEDLINE and IBECS, with the keywords validity, reproducibility and food frequency questionnaire, using the logical operator AND. Results: 15 studies were identified, from 52 to 222 food items, of quantitative type predominantly. All articles tested the validity of FFQs with 2-16 FR24H, 11 tested the reproducibility, attaining correlation coefficients between 0.4 and 0.7 in five studies, $>0.7$ in two studies, $<0.4$ in four studies, and the others between wider range of $<0.4$ to $>0.7$. The sample size varied from 40 to 499 individuals. Eight articles reported that at least one FR24H applied corresponded to at least one weekend day. In this review, were found fifteen articles on FFQ validation with the reference method FR24H. Conclusions: All had satisfactory results for validity, as suggested by the authors as a useful tool in epidemiological studies where participants have the same profile for which it was built for the FFQ.

Key words: Validity. Food Consumption 24-hour recall. Questionnaire. Adult. Elderly.

\section{Introdução}

A investigação da dieta como fator de risco para DCNT exige instrumentos de medição apropriados. ${ }^{1}$ Entre os vários métodos para avaliar a ingestão dietética, o questionário de frequência alimentar (QFA) é um instrumento com diversas vantagens sobre outros métodos, como seu baixo custo e a facilidade de uso. ${ }^{2}$ Além disso, ele pode mensurar padrões de consumo alimentar e, sobretudo, permite uma avaliação da ingestão alimentar referente a um período prolongado de tempo, uma observação importante em estudos de coorte. ${ }^{3,4}$

No entanto, as informações coletadas por um QFA são geralmente menos precisas em comparação a outros métodos, tais como o autorregistro alimentar (ARA) ou o inquérito dietético recordatório de 24 horas (IDR24H). ${ }^{2}$ Ao se adotar o QFA para avaliar o consumo alimentar em populações, é fundamental que ele contemple itens alimentares ou preparações habituais daquela população. Erros de medição podem afetar adversamente os resultados decorrentes de estudos 
que examinam a associação entre dieta e doença. ${ }^{5}$ Dessa forma, QFAs recém-desenvolvidos ou adaptados para a população foco do estudo precisam ser validados para garantir a precisão na avaliação da ingestão alimentar da população de interesse, ${ }^{6}$ bem como precisam ser avaliados em termos de sua reprodutibilidade. O termo "validade" é geralmente definido como o grau em que um instrumento mede o que se propõe a medir, ${ }^{7,8}$ diz da sua acurácia, verdade; já a reprodutibilidade verifica se os resultados são similares em duas ou mais ocasiões distintas de mensuração ${ }^{9}$ - referese portanto à sua precisão, concordância.

A validação e a avaliação da reprodutibilidade são procedimentos considerados críticos para o sucesso do QFA em medir com precisão a ingestão dietética em subgrupos da população. ${ }^{4} \mathrm{O}$ uso de listas inadequadas de alimentos no QFA pode resultar em subestimação do consumo de nutrientes, devido à omissão de elementos essenciais ${ }^{10}$ ou em superestimação, em função de indução da resposta por um número extensivo de itens alimentares. Uma técnica amplamente utilizada para validação do QFA é a comparação com métodos de referência, tais como biomarcadores, registros dietéticos e/ou múltiplos IDR24H. ${ }^{6}$ No entanto, nenhum desses métodos é capaz de medir o consumo real sem limitações, ou seja, não existe um padrão ouro.

Embora os biomarcadores sejam mais objetivos do que outros métodos, eles são geralmente caros, validam apenas um nutriente específico e constituem um método invasivo quando comparados aos inquéritos dietéticos. ${ }^{11}$ Por estas razões, o registro dietético tem sido sugerido como o método de escolha para validação de QFA; ${ }^{12}$ além disso, seu erro de estimação não é geralmente correlacionado com os erros cometidos no QFA.

Múltiplos recordatórios de $24 \mathrm{~h}$ são mais propensos a se correlacionar com os erros observados com o QFA, como o viés de memória. No entanto, a velocidade e facilidade de administração, o baixo custo e a viabilidade para entrevistar um grande número de indivíduos tornam o IDR24H um método de referência em estudos de validação de QFA. ${ }^{11}$ Assim, QFAs são geralmente validados com múltiplos recordatórios de $24 \mathrm{~h} .{ }^{13}$

Métodos para avaliar e interpretar a validade e a reprodutibilidade do QFA se utilizam da análise de correlação de nutrientes e/ou alimentos medidos por dois ou mais métodos de avaliação dietética, como os coeficientes de correlação (tais como Pearson, utilizado para analisar a validade entre dados simétricos; Spearman, para analisar a validade entre dados assimétricos; e Intraclasse, para analisar a reprodutibilidade). Uma abordagem alternativa tem sido aplicada como o método de Bland-Altman, ${ }^{14}$ que avalia a concordância entre os métodos. ${ }^{15}$ Em muitos estudos epidemiológicos, no entanto, a preocupação principal é classificar os indivíduos em diferentes grupos, de acordo com os níveis de exposição, em vez de avaliar sua ingestão absoluta. Assim, comparações de acordo com o percentual de quartil na distribuição também são frequentemente utilizadas na avaliação da validade e reprodutibilidade de um QFA. ${ }^{15}$ 
Embora a contribuição de fatores dietéticos sobre o desenvolvimento de doenças crônicas, especialmente câncer e doenças cardiovasculares, venha sendo cada vez mais reconhecida, a medição da ingestão alimentar habitual continua sendo uma das tarefas mais desafiadoras em epidemiologia nutricional. ${ }^{16}$ Nesse contexto, os estudos de validação de QFA são fundamentais para serem reconhecidos como ferramenta capaz de medir o que se propõe.

Partindo destas considerações, a presente revisão tem como objetivo descrever os estudos de validade de QFAs elaborados para população adulta e/ou idosa cujo padrão de referência seja o inquérito dietético recordatório de 24 horas (IDR24H).

\section{Métodos}

\section{Delineamento e critérios de seleção}

O presente estudo é uma revisão sistemática da literatura na qual foram incluídos artigos originais que testaram a validade (com e sem avaliação de reprodutibilidade) de questionários de frequência alimentar elaborados para população adulta ou idosa, tendo como padrão de referência o IDR24H. Foram excluídos: artigos que utilizaram outro tipo de padrão de referência que não o IDR24H; os que não foram aplicados em entrevista presencial; os que testaram apenas a reprodutibilidade e não fizeram a avaliação da validade; os que analisaram nutrientes isolados; e aqueles cuja população alvo eram crianças, adolescentes, mulheres gestantes e puérperas e portadores de doenças. Estudos em duplicidade, assim como artigos de revisão, também foram excluídos.

\section{Sistematização de busca das referências}

Foram consultadas as bases de dados eletrônicas SciELO (Biblioteca Eletrônica Cientifica Online), PubMed (Biblioteca Nacional dos Estados Unidos de Medicina), LILACS (Latin American and Caribbean Health Sciences), IBECS (Spanish Bibliographic Index of Sciences Health) e MEDLINE (Literatura Internacional em Ciências da Saúde). Estas três últimas bases foram acessadas por meio da Biblioteca Virtual em Saúde (BVS), do Centro Latino-Americano e do Caribe de Informação em Ciências da Saúde (Bireme) / Organização Pan-Americana de Saúde (OPAS) / Organização Mundial da Saúde (OMS) (http://bases.bvs.br). A busca foi dividida em quatro etapas: 
- 1aa etapa: aplicadas as palavras-chave: validade (validity), reprodutibilidade (reproducibility) e questionário de frequência alimentar (food frequency questionnaire). Recorreu-se ao operador lógico "AND" para combinação dos descritores. Nenhum limite de busca foi ativado.

- 2⿳a etapa: leitura dos títulos para uma primeira seleção dos artigos de interesse para a revisão.

- $3^{\mathbf{a}}$ etapa: verificação de ocorrência de duplicidade dos artigos nas bases de dados, sendo descartados aqueles que se repetiram.

- 4 $4^{\mathbf{a}}$ etapa: leitura dos resumos, sendo excluídos aqueles que se enquadravam nos critérios de exclusão. Quando o resumo não apresentou informações necessárias para classificá-lo de acordo com o objetivo, foram realizadas leitura e análise do artigo por completo.

\section{Síntese e comparação dos estudos}

Os estudos incluídos na revisão foram lidos na íntegra e sintetizados de forma a permitir comparação dos dados quanto à referência; local de realização do estudo; público-alvo e número da amostra; número de itens alimentares e tipo de QFA; metodologia de aplicação; e resultado/ conclusão.

\section{Resultados}

\section{Estudos de validação de QFAs tendo como referência o IDR24H}

Foram identificadas, inicialmente, 1.318 publicações (PubMed: 803; MEDLINE: 492; LILACS: 22; Scielo: 01). Após a avaliação dos títulos, 130 artigos foram considerados elegíveis, dos quais foram excluídos aqueles em duplicidade e que se enquadravam nos critérios de exclusão, resultando em 69 artigos, submetidos à $4^{\mathrm{a}}$ etapa, dos quais restaram 22 artigos a partir da leitura dos resumos; e por fim, 15 artigos após a leitura dos estudos por completo, que perfizeram o universo da presente revisão sistemática.

A tabela 1 ilustra uma síntese das principais características dos trabalhos selecionados. 
Tabela 1. Estudos de reprodutibilidade e validação de QFA com IDR24H como padrão de referência. Maceió-AL, 2011.

\begin{tabular}{|c|c|c|c|c|}
\hline Referência & $\begin{array}{c}\text { População-Alvo / } \\
\text { N / } \\
\text { Idade }\end{array}$ & $\begin{array}{c}\text { N Itens } \\
\text { alimentares / Tipo } \\
\text { de QFA }\end{array}$ & $\begin{array}{l}\text { Metodologia } \\
\text { de aplicação }\end{array}$ & $\begin{array}{l}\text { Resultado / } \\
\text { Conclusão }\end{array}$ \\
\hline $\begin{array}{l}\text { Marques- } \\
\text { Vidal } \text { et al. }{ }^{2}\end{array}$ & $\begin{array}{c}\text { Adultos suíços de } \\
\text { língua francesa } \\
23 \text { homens e } 17 \\
\text { mulheres } \\
25-65 \text { anos }\end{array}$ & $\begin{array}{c}126 \\
\text { quantitativo }\end{array}$ & $\begin{array}{c}2 \text { IDR } 24 \mathrm{H} \text { e } 2 \\
\text { QFA. }\end{array}$ & $\begin{array}{l}\text { Instrumento válido } \\
\text { para determinação } \\
\text { de macronutrientes } \\
\text { com boa } \\
\text { reprodutibilidade. }\end{array}$ \\
\hline $\begin{array}{l}\text { Pakseresht } \\
\text { et al. }{ }^{6}\end{array}$ & $\begin{array}{l}\text { Adultos canadenses } \\
12 \text { homens e } 48 \\
\text { mulheres } \\
\text { homens } \approx 46 \text { anos e } \\
\text { mulheres } \approx 45 \text { anos }\end{array}$ & $\begin{array}{c}142 \\
\text { quantitativo }\end{array}$ & $\begin{array}{c}3 \text { IDR24H e } 1 \\
\text { QFA. }\end{array}$ & QFA válido. \\
\hline $\begin{array}{l}\text { Turconi } \\
\text { et al. }{ }^{24}\end{array}$ & $\begin{array}{c}\text { Adultos italianos } \\
52 \text { homens e } 60 \\
\text { mulheres } \\
39,4 \pm 12,7 \text { anos }\end{array}$ & $\begin{array}{c}189 \\
\text { quantitativo }\end{array}$ & $\begin{array}{l}4 \text { IDR24H (com } \\
\text { intervalo de } 6 \\
\text { semanas) e } 2 \text { QFA } \\
\text { (com intervalo } 6 \\
\text { semanas). }\end{array}$ & $\begin{array}{l}\text { Confiabilidade e } \\
\text { validade relativa } \\
\text { boa. }\end{array}$ \\
\hline $\begin{array}{l}\text { Carithers } \\
\text { et al. }{ }^{1}\end{array}$ & $\begin{array}{c}\text { População adulta } \\
\text { sul-africana dos } \\
\text { EUA } \\
499 \text { homens e } \\
\text { mulheres } \\
\text { 35-81 anos }\end{array}$ & $\begin{array}{c}158 \\
\text { quantitativo }\end{array}$ & $\begin{array}{c}4 \text { IDR24H e } 1 \\
\text { QFA. }\end{array}$ & $\begin{array}{l}\text { Válido e } \\
\text { culturalmente } \\
\text { sensível }\end{array}$ \\
\hline $\begin{array}{l}\text { Wang } \\
\text { et al. }{ }^{25}\end{array}$ & $\begin{array}{l}\text { Chineses - } \\
125 \text { homens e } \\
\text { mulheres } \\
50-80 \text { anos }\end{array}$ & $\mathrm{NI}^{* *}$ & $\begin{array}{l}4 \text { IDR24H (com } \\
\text { intervalo de } 3 \\
\text { meses) e } 2 \text { QFA } \\
\text { (com intervalo de } \\
\text { um ano). }\end{array}$ & $\begin{array}{l}\text { Razoável validade e } \\
\text { reprodutibilidade. }\end{array}$ \\
\hline
\end{tabular}




\begin{tabular}{|c|c|c|c|c|}
\hline Referência & $\begin{array}{c}\text { População-Alvo / } \\
\text { N / } \\
\text { Idade }\end{array}$ & $\begin{array}{c}\text { N Itens } \\
\text { alimentares / Tipo } \\
\text { de QFA }\end{array}$ & $\begin{array}{l}\text { Metodologia } \\
\text { de aplicação }\end{array}$ & $\begin{array}{l}\text { Resultado / } \\
\text { Conclusão }\end{array}$ \\
\hline $\begin{array}{l}\text { Zanolla } \\
\text { et al. }{ }^{9}\end{array}$ & $\begin{array}{l}\text { Adultos residentes } \\
\text { em Porto Alegre, } \\
\text { Rio Grande do Sul } \\
31 \text { homens e } 52 \\
\text { mulheres } \\
41 \pm 3,42 \text { anos }\end{array}$ & $\begin{array}{c}127 \\
\text { quantitativo }\end{array}$ & $\begin{array}{c}3 \text { IDR24H e } 2 \\
\text { QFA. }\end{array}$ & $\begin{array}{l}\text { Reprodutibilidade } \\
\text { satisfatória para } \\
\text { todos os nutrientes, } \\
\text { com exceção } \\
\text { do carboidrato, } \\
\text { e validade } \\
\text { relativa razoável, } \\
\text { especialmente para } \\
\text { os macronutrientes } \\
\text { e vitamina C. }\end{array}$ \\
\hline $\begin{array}{l}\text { Sudha } \\
\text { et al. } .^{23}\end{array}$ & $\begin{array}{c}\text { Adultos do sul da } \\
\text { Índia } \\
102 \\
\geq 20 \text { anos }\end{array}$ & $\begin{array}{c}222 \\
\text { semiquantitativo. }\end{array}$ & $\begin{array}{l}2 \text { IDR24H (com } \\
\text { intervalo de } 2 \\
\text { meses) e } 3 \text { QFA } \\
\text { (0,6 e 12meses). }\end{array}$ & $\begin{array}{l}\text { Ferramenta robusta } \\
\text { para avaliar } \\
\text { consumo alimentar. }\end{array}$ \\
\hline $\begin{array}{l}\text { Ribeiro } \\
\text { et al. }{ }^{21}\end{array}$ & $\begin{array}{c}\text { Adultos - } \\
38 \text { homens e } 37 \\
\text { mulheres } \\
35,4 \pm 11,8 \text { anos }\end{array}$ & $\begin{array}{c}52 \\
\text { semiquantitativo. }\end{array}$ & $\begin{array}{c}3 \text { IDR24H e } 2 \\
\text { QFA }\end{array}$ & $\begin{array}{l}\text { Bom Instrumento } \\
\text { de pesquisa. }\end{array}$ \\
\hline $\begin{array}{l}\text { Block } \\
\text { et al. }{ }^{33}\end{array}$ & $\begin{array}{l}\text { Hispânicos de } \\
\text { baixa renda - } \\
37 \text { homens e } 52 \\
\text { mulheres } \\
\approx 36,8 \text { anos }\end{array}$ & $\begin{array}{c}103 \\
\text { quantitativo. }\end{array}$ & $\begin{array}{c}3 \text { IDR24H e } 1 \\
\text { QFA }\end{array}$ & Validade razoável. \\
\hline $\begin{array}{l}\text { Kusama } \\
\text { et al. } .^{20}\end{array}$ & $\begin{array}{c}56 \text { homens e } 62 \\
\text { mulheres } \\
\text { 23-62 anos }\end{array}$ & $\begin{array}{c}116 \\
\text { quantitativo. }\end{array}$ & $\begin{array}{c}3 \text { IDR24H e } 2 \\
\text { QFA }\end{array}$ & $\begin{array}{l}\text { Ferramenta } \\
\text { adequada para } \\
\text { avaliar a ingestão } \\
\text { dietética para } \\
\text { a maioria dos } \\
\text { nutrientes. }\end{array}$ \\
\hline
\end{tabular}




\begin{tabular}{|c|c|c|c|c|}
\hline Referência & $\begin{array}{c}\text { População-Alvo / } \\
\text { N / } \\
\text { Idade }\end{array}$ & $\begin{array}{c}\text { N Itens } \\
\text { alimentares / Tipo } \\
\text { de QFA }\end{array}$ & $\begin{array}{l}\text { Metodologia } \\
\text { de aplicação }\end{array}$ & $\begin{array}{l}\text { Resultado / } \\
\text { Conclusão }\end{array}$ \\
\hline $\begin{array}{l}\text { Fornés } \\
\text { et al. }{ }^{17}\end{array}$ & $\begin{array}{l}\text { Trabalhadores } \\
\text { brasileiros de } \\
\text { baixa renda e } \\
\text { alfabetização } \\
42 \text { homens e } 62 \\
\text { mulheres } \\
18-60 \text { anos }\end{array}$ & $\begin{array}{c}127 \\
\text { qualitativo. }\end{array}$ & $\begin{array}{l}6 \text { IDR24H (entre } \\
\text { período de } 6 \\
\text { meses entre os } \\
\text { QFAs) e } 2 \text { QFA } \\
\text { (entre período de } \\
6 \text { meses). }\end{array}$ & $\begin{array}{l}\text { Satisfatória } \\
\text { reprodutibilidade e } \\
\text { razoável validade. }\end{array}$ \\
\hline $\begin{array}{l}\text { Rodríguez } \\
\text { et al. } .^{29}\end{array}$ & $\begin{array}{l}\text { População da zona } \\
\text { leste da Guatemala } \\
73 \text { indivíduos } \\
22-55 \text { anos }\end{array}$ & $\begin{array}{c}52 \\
\text { semiquantitativo }\end{array}$ & $\begin{array}{c}\text { 2-3 IDR24H e } 1 \\
\text { QFA }\end{array}$ & Ferramenta útil. \\
\hline $\begin{array}{l}\text { Salvo e } \\
\text { Gimeno }^{22}\end{array}$ & $\begin{array}{l}\text { Adultos com } \\
\text { excesso de peso } \\
146 \text { indivíduos } \\
\text { 18-60 anos }\end{array}$ & $\begin{array}{c}90 \\
\text { quantitativo. }\end{array}$ & $\begin{array}{c}3 \text { IDR24H (com } \\
\text { intervalo de } 15 \\
\text { dias) e } 2 \text { QFA } \\
\text { (com intervalo de } \\
\text { 47dias). }\end{array}$ & $\begin{array}{l}\text { Boa validade e } \\
\text { reprodutibilidade. }\end{array}$ \\
\hline $\begin{array}{l}\text { Jackson } \\
\text { et al. }{ }^{19}\end{array}$ & $\begin{array}{c}\text { Adultos jamaicanos } \\
\text { de origem africana } \\
\text { Para } \\
\text { reprodutibilidade: } \\
123 \text { e para a } \\
\text { validade: } 73 \\
\text { indivíduos } \\
25-74 \text { anos }\end{array}$ & $\begin{array}{c}70 \\
\text { quantitativo. }\end{array}$ & $\begin{array}{c}12 \text { IDR24H e } 2 \\
\text { QFA }\end{array}$ & $\begin{array}{l}\text { Reprodutibilidade } \\
\text { razoável e adequada } \\
\text { validade. }\end{array}$ \\
\hline $\begin{array}{l}\text { Hernández- } \\
\text { Avila et al. }{ }^{18}\end{array}$ & $\begin{array}{c}\text { Mulheres } \\
\text { mexicanas de baixo } \\
\text { nível econômico } \\
134 \text { mulheres. } \\
\text { NI** }^{* *}\end{array}$ & $\begin{array}{c}116 \\
\text { semiquantitativo. }\end{array}$ & $\begin{array}{c}16 \text { IDR24H e } 2 \\
\text { QFA }\end{array}$ & $\begin{array}{l}\text { QFA útil para } \\
\text { avaliar ingestão } \\
\text { dietética. }\end{array}$ \\
\hline
\end{tabular}

N: Tamanho da amostra; NI: Não Informado; QFA: Questionário de Frequência Alimentar; IDR24H: Inquérito dietético recordatório de 24 horas; NI: Não Informado. 


\section{Considerações metodológicas acerca da validação e reprodutibilidade} de QFAs apresentadas nesta revisão

Dos 15 artigos selecionados que testaram a validade de QFAs, 11 (73,33\%) analisaram também a sua reprodutibilidade., ${ }^{2,917-25}$

O tamanho da amostra estudada variou entre $40^{2}$ e 499 indivíduos. ${ }^{1}$ Para a validação de um instrumento, recomenda-se uma amostra de 100 a 200 pessoas, ${ }^{26,27}$ pois estudos com mais de 200 pessoas contribuiriam com pouca precisão para se ter um intervalo de confiança correto. Neste intervalo de " $\mathrm{n}$ " se enquadraram 46,66\% (n=7) dos 15 estudos revisados e apenas 6,66\% (n=1) trabalharam com amostra acima de 200. Em contrapartida, estudos com menos de 30 pessoas aumentariam a amplitude do intervalo de confiança, ${ }^{26,27} \mathrm{o}$ que não se aplica aos estudos incluídos nesta revisão. Mais recentemente, tem sido recomendada uma amostra de 50 a 100 pessoas para cada grupo demográfico ${ }^{28}$ como um número apropriado para validação, o que foi identificado em $40 \%$ $(\mathrm{n}=6)$ dos estudos investigados. A idade dos indivíduos também é um fator que merece destaque na delimitação da amostra. Apesar de todas as populações serem compostas por adultos, algumas faixas etárias foram mais amplas, incluindo idosos. Segundo a literatura, a idade do entrevistado também pode influenciar a fidedignidade das informações colhidas pelo QFA, ${ }^{21}$ provavelmente por questões de memória e cooperação por parte dos entrevistados.

Quanto ao número de itens alimentares, os QFAs analisados apresentaram entre $52^{21,29} \mathrm{e} 222^{23}$ itens. QFAs com listas alimentares curtas (menos de 50 itens) não avaliam corretamente a ingestão habitual, e listas extensas (com mais de 100 itens alimentares) comprometem a vantagem de rapidez e simplicidade do QFA. ${ }^{30}$ Considerando que na construção de um QFA listas longas superestimam a ingestão, enquanto que listas pequenas a subestimam, ${ }^{26} \mathrm{o}$ número de itens alimentares presente no QFA deve ser adequado, respeitando os limites referidos, para não se tornar cansativo e, ao mesmo tempo, ser capaz de mensurar o que o QFA se propõe. Na presente revisão, 73,33\% (n=11) dos QFAs analisados apresentavam uma lista com mais de 100 itens alimentares e 26,66\% ( $\mathrm{n}=4)$ possuíam uma lista entre 50 e 100 itens alimentares.

Outro aspecto importante no que se refere às questões metodológicas é o momento da aplicação. É recomendável que o QFA seja aplicado sempre antes do método de referência (ARA ou IDR24H), mesmo que este seja aplicado imediatamente após, por dois motivos: primeiro, porque os indivíduos participantes da pesquisa não passaram por qualquer outra avaliação dietética que poderia influenciar no procedimento de validação; segundo, porque o ato de preencher os formulários de referência poderá direcionar a atenção a suas próprias dietas. ${ }^{3}$ Willett $^{27}$ sugere que a administração do QFA seja realizada juntamente com o método de referência. 
O número de dias de informações dietéticas depende dos parâmetros dietéticos a serem considerados, da extensão da variabilidade na população, dos objetivos da pesquisa e da variabilidade dos nutrientes ou alimentos a serem medidos. ${ }^{26}$ Recomendam-se no mínimo dois e no máximo 28 dias de medições do método de referência para um estudo de validação; ${ }^{31}$ contudo, Rosner e Willet ${ }^{32}$ defendem que, quando são utilizadas poucas medidas, é necessário um ajuste estatístico para a remoção dos efeitos de variação intrapessoal. Na presente revisão 13,33\% (n=2) aplicaram dois IDR24H; 46,66\% ( $\mathrm{n}=7)$ três; 26,66\% $(\mathrm{n}=4)$ aplicaram de quatro a seis IDR24H e 13,33\% ( $\mathrm{n}=2)$ mais de 12 IDR24H, para testar a validade dos QFA estudados - ou seja, 100\% das pesquisas seguiram a recomendação de aplicação de IDR24H pelo menos duas vezes e, ainda, mais de 82,35\% aplicaram o IDR24H mais de três vezes, o que revela uma preocupação em medir adequadamente o consumo alimentar.

Além do número de aplicações, é importante atentar para o dia da semana ao qual o IDR24H se reporta. Sugere-se que um dos dias avaliados corresponda a um dia de final de semana, pois representa um dia de consumo geralmente diferente dos outros dias da semana. ${ }^{7,26}$ Como foi referido em 47,05\% $(n=8)$ dos artigos, pelo menos um dos IDR24H correspondia a um dia de final de semana. Esse percentual abaixo da metade dos artigos estudados é preocupante, uma vez que o fato de não inserir um dia de final de semana pode comprometer o resultado da pesquisa em termos de subestimar os valores encontrados pelos IDR24H, o que prejudicaria a validade encontrada.

Diferentemente do encontrado em estudos onde as medições são feitas sob condições laboratoriais altamente controladas, estudos de reprodutibilidade de inquéritos dietéticos frequentemente apresentam valores de coeficientes de correlação entre 0,5 e $0,7 .{ }^{27}$ Willett afirma que os coeficientes de correlação devem se encontrar na faixa de 0,4 a 0,7 , para uma validação aceitável (coeficientes mais baixos do que os de medidas laboratoriais), o que foi verificado em grande parte dos estudos selecionados nesta revisão, quanto aos resultados acerca da validade e da reprodutibilidade (tabela 2). Os coeficientes de correlação obtidos pelos estudos aqui revisados na análise da validade variaram bastante em relação ao valor de referência $(0,4$ a 0,7$))^{1,9,23,25}$ Ficaram em torno desta faixa 11 estudos, assim distribuídos: 33,33\% (n=5) com valores de correlação entre 0,4 e $0,7 \cdot 3^{17,18,21,29,33} 26,66 \%(n=4)$ com valores abaixo de 0,$4 ;^{2,6,62,22}$ e apenas $13,33 \%(n=2)$ com correlação acima de $0,7 .{ }^{9,24}$ Os demais $(n=4)$ apresentaram valores de correlação dentro de uma faixa mais ampla, que foi de $<0,4$ até $>0,77^{1,19,23,25}$ apresentando estes extremos da faixa no mesmo estudo (p. ex. CC variando de 0,2 a 0,8 ). 
Tabela 2. Coeficientes de correlação da validade (V) e reprodutibilidade (R) dos Questionários de Frequência Alimentar (QFA) analisados nos artigos estudados. Maceió-AL, 2011.

\begin{tabular}{|c|c|c|}
\hline Referência & Metodologia/Estatística & Resultado \\
\hline $\begin{array}{l}\text { Marques- } \\
\text { Vidal } \text { et } a l .{ }^{2}\end{array}$ & $\begin{array}{l}\text { V: } 2 \text { IDR } 24 \mathrm{H} \text { e } 2 \text { QFAs } \\
\text {-.CCP. } \\
\text { R: } 2 \text { QFAs - CCP, CCI e } \\
\text { CCL. }\end{array}$ & $\begin{array}{l}\text { V: CCP entre IDR } 24 \mathrm{H}-\text { QFA1 }(0,31 \text { a } 0,49) \text { e } \\
\text { IDR24H - QFA2 }(0,38 \text { a } 0,59) . \\
\text { R: CCP }(0,58 \text { a } 0,9) \text { e CCI }(0,53 \text { a } 0,98) \text { e CCL }(0,55 \\
\text { a } 0,87) .\end{array}$ \\
\hline $\begin{array}{l}\text { Pakseresht } \\
\text { et al. }{ }^{6}\end{array}$ & $\begin{array}{l}\text { V: } 3 \text { IDR24H e } 1 \text { QFA } \\
\text { - CCS. }\end{array}$ & $\begin{array}{l}\text { V: CCS entre IDR24H - QFA }(0,32 \text { a } 0,35) \text {. CCS } \\
\text { deatenuado entre IDR24H - QFA }(0,33 \text { a } 0,53) .\end{array}$ \\
\hline $\begin{array}{l}\text { Turconi } \\
\text { et al. }{ }^{24}\end{array}$ & $\begin{array}{l}\text { V: } 4 \text { IDR24H E } 2 \text { QFAs } \\
\text { - CCP. } \\
\text { R: } 2 \text { QFAs - CCP. }\end{array}$ & $\begin{array}{l}\text { V: CCP entre IDR } 24 \mathrm{H}-\text { QFA }(0,7 \text { a } 0,93) \text {. } \\
\text { R: CCP entre QFA }(0,78 \text { a } 0,87) \text {. }\end{array}$ \\
\hline $\begin{array}{l}\text { Carithers } \\
\text { et al. }{ }^{1}\end{array}$ & $\begin{array}{l}\text { V: } 4 \text { IDR24H e } 1 \text { QFA } \\
\text { - CCP. }\end{array}$ & $\begin{array}{l}\text { V: Homens CCP entre IDR24H - QFA curto } \\
(\mathrm{M}=0,42) \text { e IDR24H - QFA longo }(\mathrm{M}=0,49) \text {. } \\
\text { Mulheres CCP entre IDR24H - QFA curto }(\mathrm{M}=0,31) \\
\text { e IDR24H - QFA longo }(\mathrm{M}=0,42) \text {. } \\
\text { CCP ajustado e deatenuado: IDR24H - QFA curto } \\
(0,20 \text { a } 0,70) \text { e IDR24H - QFA longo }(0,23 \text { a } 0,75) \text {. }\end{array}$ \\
\hline $\begin{array}{l}\text { Wang } \\
\text { et al. }{ }^{25}\end{array}$ & $\begin{array}{l}\text { V: } 4 \text { IDR } 24 \text { H e } 2 \text { QFAs } \\
\text { - CCS. } \\
\text { R: } 2 \text { QFAs - CCS. }\end{array}$ & $\begin{array}{l}\text { V: CCS entre IDR } 24 \mathrm{H} \text { - QFAs }(0,12 \text { a } 0,74) \text {. CCS } \\
\text { corrigido entre IDR } 24 \mathrm{H} \text { - QFAs }(0,17 \text { a } 0,79) . \\
\text { R: CCS entre QFA1 - QFA2 }(0,38 \text { a } 0,74) .\end{array}$ \\
\hline $\begin{array}{l}\text { Zanolla } \\
\text { et al. } .^{9}\end{array}$ & $\begin{array}{l}\text { V: } 3 \text { IDR24H e } 2 \text { QFAs } \\
\text { - CCI. } \\
\text { R: } 2 \text { QFAs - CCI. }\end{array}$ & $\begin{array}{l}\text { V: CCI entre IDR24H - QFA1 }(0,23 \text { a } 0,86) \text { e } \\
\text { IDR24H - QFA2 }(0,50 \text { a 0,95). } \\
\text { R: CCI entre QFAs }(0,44 \text { a } 0,83) .\end{array}$ \\
\hline $\begin{array}{l}\text { Sudha } \\
\text { et al. }{ }^{23}\end{array}$ & $\begin{array}{l}\text { V: } 2 \text { IDR } 24 \mathrm{H} \text { e } 3 \text { QFAs } \\
\text { - CCP. } \\
\text { R: } 3 \text { QFAs - CCP e CCI. }\end{array}$ & $\begin{array}{l}\text { V: CCP entre IDR } 24 \mathrm{H} \text { e QFA1 }(0,22 \text { a } 0,72) \text { e } \\
\text { IDR } 24 \mathrm{H} \text { e QFA3 }(0,32 \text { a } 0,70) \text {. CCP deatenuado entre } \\
\text { IDR } 24 \mathrm{H} \text { e QFA1 }(0,22 \text { a } 0,67) \text { e IDR } 24 \mathrm{H} \text { e QFA3 } \\
(0,35 \text { a } 0,73) \text {. } \\
\text { R: CCP entre QFAs }(0,41 \text { a } 0,86) \text { e CCI entre QFAs } \\
(0,45 \text { a } 0,72) \text {. }\end{array}$ \\
\hline
\end{tabular}




\begin{tabular}{|c|c|c|}
\hline Referência & Metodologia/Estatística & Resultado \\
\hline $\begin{array}{l}\text { Ribeiro } \\
\text { et al. } .^{21}\end{array}$ & $\begin{array}{l}\text { V: } 3 \text { IDR } 24 \text { H e } 2 \text { QFAs } \\
\text { - CCP. } \\
\text { R: } 2 \text { QFAs - CCI. }\end{array}$ & $\begin{array}{l}\text { V: CCP entre IDR24H e QFAs }(0,32 \text { a } 0,66) \text {. } \\
\text { R: CCI entre QFAs }(0,43 \text { a } 0,76) \text {. }\end{array}$ \\
\hline $\begin{array}{l}\text { Block } \\
\text { et al. }{ }^{.33}\end{array}$ & $\begin{array}{l}\text { V: } 3 \text { IDR24H e } 1 \text { QFA } \\
\text { - CCP. }\end{array}$ & $\begin{array}{l}\text { V: CCP entre IDR } 24 \mathrm{H} \text { e QFAs }(\mathrm{M}=0,52) \text { e CCP } \\
\text { deatenuado entre IDR24H e QFAs }(\mathrm{M}=0,61) .\end{array}$ \\
\hline $\begin{array}{l}\text { Kusama } \\
\text { et al. }{ }^{20}\end{array}$ & $\begin{array}{l}\text { V: } 3 \text { IDR24H e } 2 \text { QFAs } \\
\text { - CCP. } \\
\text { R: 2QFAs - CCP. }\end{array}$ & $\begin{array}{l}\text { V: CCP ajustado entre IDR24H e QFAs }(0,16 \text { a } 0,45) \text {. } \\
\text { R: CCP ajustado entre QFAs }(0,47 \text { a } 0,72) \text {. }\end{array}$ \\
\hline $\begin{array}{l}\text { Fornés } \\
\text { et al. }{ }^{17}\end{array}$ & $\begin{array}{l}\text { V: } 6 \text { IDR24H e } 2 \text { QFAs } \\
\text { - CCI. } \\
\text { R: } 2 \text { QFAs - CCP. }\end{array}$ & $\begin{array}{l}\text { V: CCI entre IDR } 24 \mathrm{H} \text { e QFAs }(0,21 \text { a } 0,70 ; \mathrm{M}=0,50) \text {. } \\
\text { CCI atenuado entre IDR24H e QFAs }(0,35 \text { a } 0,65) \text {. } \\
\text { R: CCP entre QFA1 e QFA2 }(0,23 \text { a } 0,69 ; \mathrm{M}=0,52) \text {. }\end{array}$ \\
\hline $\begin{array}{l}\text { Rodríguez et } \\
a l .{ }^{29}\end{array}$ & $\begin{array}{l}\text { V: 2-3 IDR24H e } 1 \text { QFA } \\
\text { - CCP. }\end{array}$ & $\begin{array}{l}\text { V: CCP entre IDR24H e QFA }(0,12 \text { a } 0,64) \text {. CCP } \\
\text { ajustado entre IDR24H e QFA }(0,11 \text { a } 0,59) .\end{array}$ \\
\hline $\begin{array}{l}\text { Salvo e } \\
\text { Gimeno }^{22}\end{array}$ & $\begin{array}{l}\text { V: } 3 \text { IDR24H e } 2 \text { QFAs } \\
\text { - CCI. } \\
\text { R: } 2 \text { QFAs - CCI. }\end{array}$ & $\begin{array}{l}\text { V: CCI entre IDR } 24 \mathrm{H} \text { e QFAs }(0,01 \text { a } 0,21) \text {. } \\
\text { R: CCI entre QFAs }(0,28 \text { a } 0,57) \text {. }\end{array}$ \\
\hline $\begin{array}{l}\text { Jackson } \\
\text { et al. }{ }^{19}\end{array}$ & $\begin{array}{l}\text { V: } 12 \text { IDR } 24 \mathrm{H} \text { e } 2 \text { QFAs } \\
\text { - CCP e CCI. } \\
\text { R: } 2 \text { QFAs - CCP e CCI. }\end{array}$ & $\begin{array}{l}\text { V: CCP e CCI entre IDR24H e QFAs }(0,20 \text { a } 0,86) \text {. } \\
\text { R: CCP e CGI entre QFAs }(0,42 \text { a } 0,71) \text {. }\end{array}$ \\
\hline $\begin{array}{l}\text { Hernández- } \\
\text { Avila et al. }{ }^{18}\end{array}$ & $\begin{array}{l}\text { V: } 16 \text { IDR } 24 \mathrm{H} \text { e } 2 \text { QFAs } \\
\text { - CCP. } \\
\text { R: } 2 \text { QFAs - CCP. }\end{array}$ & $\begin{array}{l}\text { V: CCP entre IDR24H e QFA1 }(0,14 \text { a } 0,51) \text {. CCP } \\
\text { ajustado entre }(0,07 \text { a } 0,49) \text {. CCP deatenuado entre } \\
(0,12 \text { a } 0,67) \text {. CCP entre IDR24H e QFA2 }(0,19 \\
\text { a } 0,57) \text {. CCP ajustado entre }(0,05 \text { a } 0,67) \text {. CCP } \\
\text { deatenuado entre }(0,12 \text { a } 0,63) \text {. } \\
\text { R: CCP entre QFAs }(0,43 \text { a } 0,60) \text {. CCP ajustado entre } \\
\text { QFAs }(0,25 \text { a } 0,50) \text {. }\end{array}$ \\
\hline
\end{tabular}

V: validade; R: reprodutibilidade; IDR24H: inquérito dietético recordatório 24 horas; QFA: questionário de frequência alimentar; CCP: coeficiente de correlação de Pearson; CCI: coeficiente de correlação intraclasse; CCL: coeficiente de correlação de Lin; CCS: coeficiente de correlação de Spearman. 
Quanto à correlação da reprodutibilidade avaliada em 11 estudos dos quinze revisados, 54,54\% $(n=6)$ apresentaram resultados com grande amplitude de variação entre as correlações encontradas, a depender do nutriente analisado;, ${ }^{918,19,21,23,25} 27,27 \%(\mathrm{n}=3)$ apresentaram valores de correlação abaixo de $0,5,{ }^{8,21,28}$ e apenas $18,18 \%$ ( $\mathrm{n}=2$ ) identificaram forte correlação para reprodutibilidade, com valores entre 0,5 e $0,7^{2,24}$

Nos artigos estudados, os QFAs, de forma geral, são classificados pelos autores como de validade boa, exceto alguns ${ }^{9,19,25,33}$ que definem o QFA avaliado como de validade razoável e boa reprodutibilidade e sugerem sua reaplicação em população-alvo similar, o que indica a necessidade da validação do instrumento.

\section{Conclusão}

Considerando os procedimentos metodológicos a serem empregados no processo de validação de QFAs aqui revisados, tais como tamanho da amostra, número de itens alimentares, hierarquia de aplicação vs método de referência, bem como número de dias de medição, pode-se concluir que a maioria dos artigos selecionados apresentou resultado satisfatório para validade e reprodutibilidade. Esses achados, obtidos em estudos com esta complexidade, permitiram que os autores os apresentassem como ferramenta útil em estudos epidemiológicos, passíveis de serem aplicados em outras populações com o mesmo perfil para o qual foi destinado o QFA de origem.

Todas as considerações metodológicas discutidas são decisivas para atingir níveis satisfatórios de validade e reprodutibilidade, atestando a natureza laboriosa desta linha de investigação.

\section{Agradecimentos}

À Coordenação de Aperfeiçoamento de Pessoal de Nível Superior (CAPES), pela concessão da bolsa de mestrado. 


\section{Referências}

1. Carithers TC, Talegawkar SA, Rowser ML, Henry OR, Dubbert PM, Bogle ML, Taylor HA, Tucker KL. Validity and calibration of food frequency questionnaires used with African-American adults in the Jackson Heart Study. J Am Diet Assoc. 2009;109(7):1184-93.

2. Marques-Vidal P, Ross A, Wynn E, Rezzi S, Paccaud F, Decarl BI. Reproducibility and relative validity of a food-frequency questionnaire for French-speaking Swiss adults. Food Nutr Res. 2011;55.

3. Nelson M, Bingham SA. Assessment of food consumption and nutrient intake. In Design Concepts in Nutritional Epidemiology. 2nd ed. B. M. New York, NY: Oxford University Press; 2003.

4. Subar AF. Developing dietary assessment tools. J Am Diet Assoc. 2004;104:769-70.

5. Fraser GE, Butler TL, Shavlik D. Correlations between estimated and true dietary intakes: Using two instrumental variables. Ann Epidemiol. 2005;15:509-18.

6. Pakseresht M, Sharma S. Validation of a culturally appropriate quantitative food frequency questionnaire for Inuvialuit population in the Northwest Territories, Canada. J Hum Nutr Diet. 2010;23(Suppl. 1):75-82.

7. Beaton G, Burema J, Ritenbaugh C. Errors in the interpretation of dietary assessments. Am J Clin Nutr. 1994;65:1100-1007.

8. Lopes ACS, Caiaffa WT, Migoti SA, Lima-Costa MFF. Ingestão alimentar em estudos epidemiológicos. Rev Bras Epidemiol. 2003;6(3):209-19.

9. Zanolla AF, Olinto MTA, Henn RL, Wahrlich V, Anjos LA. Avaliação de reprodutibilidade e validade de um questionário de frequência alimentar em adultos residentes em Porto Alegre, Rio Grande do Sul, Brasil. Cad Saúde Pública. 2009;25(4):840-8.

10. Sharma S, Cade J, Jackson M, Mbanya JC, Chungong S, Forrester T, Bennett F, Wilks R, Balkau B, Cruickshank JK. Development of food frequency questionnaires in three population samples of African origin from Cameroon, Jamaica and Caribbean migrants to the UK. Eur J Clin Nutr. 1996; 50:479-86.

11. Nelson M. The validation of dietary assessment. In Design and concepts in Nutritional Epidemiology, 2nd ed. New York, NY: Oxford University Press, 2003.

12. Cade J, Thompson R, Burley V, Warm D. Development, validation and utilisation of food-frequency questionnaires - a review. Public Health Nutr. 2002;5(4):567-87.

13. Subar AF, Ziegler RG, Thompson FE, Johnson CC, Weissfeld JL, Reding D, Kavounis KH, Hayes $\mathrm{RB}$. Is shorter always better? Relative importance of questionnaire length and cognitive ease on response rates and data quality for two dietary questionnaires. Am J Epidemiol. 2001;153:404-9.

14. Bland JM, Altman DG. Statistical methods for assessing agreement between two methods of clinical measurement. Lancet 1. 1986; 307-10. 
15. Villegas R, Yang G, Liu D, Xiang Y-B, Cai H, Zheng W, Shu XO. Validity and reproducibility of the food-frequency questionnaire used in the Shanghai Men's Health Study. Br J Nutr. 2007;97:993-1000.

16. Shu XO, Yang G, Jin F, Liu D, Kushi L, Wen W, Gao Y-T, Zheng W. Validity and reproducibility of the food frequency questionnaire used in the Shanghai Women's Health Study. Eur J Clin Nutr. 2004;58:17-23.

17. Fornés NS, Stringhini MLF, Elias BM. Reproducibility and validity of a food-frequency questionnaire for use among low-income Brazilian workers. Public Health Nutr. 2003;6(8):821-7.

18. Hernández-Avila M, Romieu I, Parra S, Hernández-Avila J, Madrigal H, Willett W. Validity and reproducibility of a food frequency questionnaire to assess dietary intake of women living in Mexico City. Salud Publica Mex. 1998;39(40).

19. Jackson M, Walker S, Cade J, Forrester T, Cruickshank JK, Wilks R. Reproducibility and validity of a quantitative food frequency questionnaire among Jamaicans of African origin. Public Health Nutr. 2001;4:971-80.

20. Kusama K, Le DSNT, Hanh TTM, Takahashi K, Hung NTK, Yoshiike N, Yamamoto S. Reproducibility and validity of a food frequency questionnaire among Vietnamese in Ho Chi Minh City. 2005;24(6):466-73.

21. Ribeiro AC, Sávio KEO, Rodrigues MLCF, Costa THM, Schmitz BAS. Validação de um questionário de frequência de consumo alimentar para população adulta. Rev Nutr. 2006;19(5):553-62.

22. Salvo VLMA, Gimeno SGA. Reprodutibilidade e validade do questionário de frequencia de consumo de alimentos. Rev Saúde Pública. 2002;36(4):505-12.

23. Sudha G, Radhika RM, Sathya AG, Mohan V. Reproducibility and validity of an interviewer administered semi-quantitative food frequency questionnaire to assess dietary intake of urban adults in southern India. Int J Food Sci Nutr. 2006;57(7/8):481-93.

24. Turconi G, Bazzano R, Roggi C, Cena H. Reliability and relative validity of a quantitative foodfrequency questionnaire for use among adults in Italian population. 2010;61(8): 846-62.

25. Wang X, Yan H, Sa R. Study of validity and reproducibility of food frequency questionnaires for residents over 50-years-old in Xi’an City. Wei Sheng Yan Jiu. 2009 May; 38(3):299-302.

26. Thompson FE, Byers T. Dietary assessment resource manual. Journal of Nutrition. 1994;124:2245S-2317S.

27. Willett W. Nutritional Epidemiology. 2nd ed. New York: Oxford University Press; 1998.

28. Burley V, Cade J. Consensus Documento on the development, validation and utilization of food frequency questionnaires. The fourth International Conference on Dietary Assessment Methods. Tucson, Arizona (USA), 2000.

29. Rodriguez MM, Mendez H, Torun B, Schroeder D, Stein AD. Validation of a semi-quantitative foodfrequency questionnaire for use among adults in Guatemala. Public Health Nutr. 2002;5(5):691-8. 
30. Fisberg RM, Slater B, Marchioni DML, Martini LA. Inquéritos alimentares: métodos e bases científicas. Barueri: Manole; 2005.

31. Slater B, Philippi ST, Fisberg RM, Latorre MRDO. Validation of a semiquantitative adolescent food frequency questionnaire applied at a public school in São Paulo, Brazil. Eur J Clin Nutr. 2003;57:629-35.

32. Rosner B, Willett W. Interval estimates for correlation coefficients corrected for within-person variation: implications for study design and hypothesis testing. Am J Epidemiol. 1988;127:377-86.

33. Block G, Wakimoto P, Jensen C, Mandel S, Green RR. Validation of a food frequency questionnaire for hispanics. 2006; 3(3):1-10.

Recebido: 30/4/2013

Revisado: 25/7/2013

Aprovado: 30/7/2012 